\title{
Von Grund auf Spitze
}

\begin{abstract}
«Welche Hausarztmedizin braucht die Spitzenmedizin, welche Spitzenmedizin die Hausarztmedizin?» - An diesen Leitfragen orientierte sich das 6. Platin-Symposium der SGIM. Auch wenn endgültige Antworten erwartungsgemäss ausblieben, wurden in anregenden Referaten und Diskussionen der Platin-Tradition entsprechend überraschende Perspektiven aufgezeigt und breitgefächerte Impulse vermittelt.
\end{abstract}

Bruno Kesseli

bkesseli@emh.ch
«Spitzenmedizin» und «Grundversorgung», wie die Hausarztmedizin seit einigen Jahren in Fachkreisen bevorzugt genannt wird, bilden schon begrifflich ein spannungsreiches Gegensatzpaar. Zwar lässt sich Grundversorgung assoziativ in eine Reihe stellen mit bedeutungsschweren Begriffen wie Grundbedürfnisse, Grundwerte oder Grundlagen, was ihre grundlegende Bedeutung und zentrale Stellung in der Medizin verdeutlichen mag. In der direkten Gegenüberstellung mit der Spitzenmedizin, die bildlich gewissermassen auf dem Olymp der ärztlichen Künste thront, haftet der Grundversorgung konnotativ indessen etwas «Bodensatzmässiges», Niedriges, vielleicht sogar Unappetitliches an. «Grundversorger - was ist das für ein Wort?», fragte der Philosoph und Publizist Ludwig Hasler im Abschlussreferat des diesjährigen Platin-Symposiums denn auch mit innerem Kopfschütteln, um zu ergänzen: «Bitte unterschätzen Sie die Sprache nicht.»
Werturteile, Haltungen, Einstellungen (auch kollektive), schwer fassbare Grössen wie der «Zeitgeist» manifestieren sich eben auch - vielleicht sogar in erster Linie - in der Sprache. Dass die Assoziationen in der französischsprachigen Schweiz durch den Begriff «médecine de premier recours» in eine andere Richtung gelenkt werden als in der Deutschschweiz, wie ein Teilnehmer französischer Muttersprache bemerkte, ist ein sprachkulturelles Phänomen, das einer gesonderten Betrachtung würdig wäre.

\section{Von Spitzenköchen und Spitzenmedizinern}

$\mathrm{Ob}$ es an derartigen sprachlichen Erwägungen der Organisatoren lag, dass die Modewörter «Grundversorger» und «Grundversorgung» im Symposiumsprogramm nicht vertreten waren, sondern durchwegs die gute alte «Hausarztmedizin» zum Zug kam? Letztere blieb zumindest als Begriff unbehelligt, während ihr
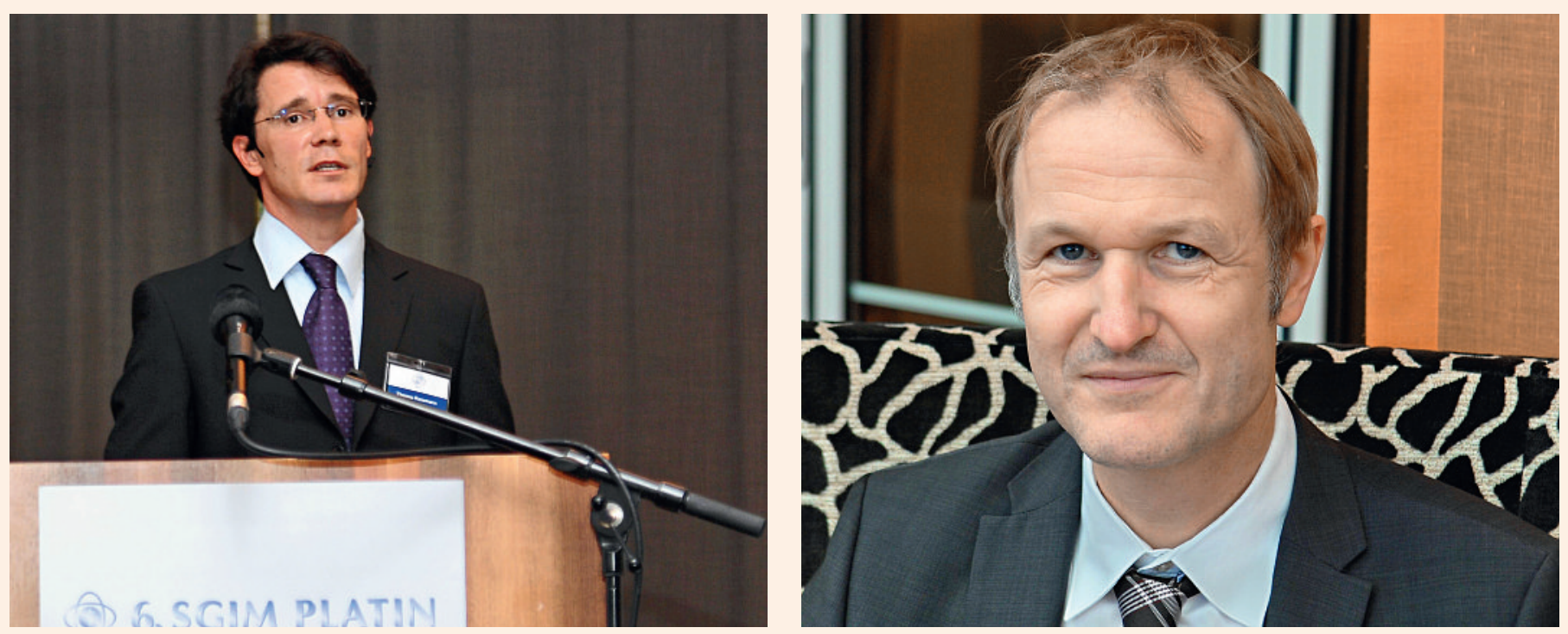

Vertraten die Sicht der Hausarzt- bzw. der hochspezialisierten Medizin: Thomas Rosemann (links) und Volkmar Falk. Fotos: Heidi Fuchs, Healthworld (Schweiz) AG. 
Konterpart, die Spitzenmedizin, Philosophen und Mediziner definitorisch gleichermassen herausforderte. «Was ist Spitzenmedizin?» - eine Frage, die an diesem Anlass wie bereits an vielen anderen mehrfach gestellt und mit einer beträchtlichen Bandbreite beantwortet wurde. Einige Positionen:

- «Spitze» können die Quantität, die Qualität, die Innovation, die Spezialisierung, die Kosten sein. Im Rahmen der Verteilungsfrage sind die Spezialisierung und die Kosten der Spitzenmedizin von primärer Bedeutung. Die Spitzenmedizin fokussiert auf Teile des Körpers und ist in der Regel kostenintensiv (Dr. phil. Richard Egger, Philosoph und Unternehmensberater).
Pasta auskenne und Kochkunst in Vollendung praktiziere, müsste der «Spitzenmediziner» der Medizin in einem umfassenden Sinn gerecht werden. In der sogenannten Spitzenmedizin seien in diesem Sinn nicht unbedingt Spitzenärzte tätig eher Stars, Virtuosen hochspezialisierter Technik. Im Rahmen des Symposiums erwies sich die deskriptive Auffassung der Spitzenmedizin als einer hochspezialisierten, kostenintensiven Medizin aus pragmatischen Gründen dann doch als die tauglichste. So einleuchtend und berechtigt - jede Ärztin, jeder Arzt kann «spitze» sein - der qualitative Ansatz ist: Wenn potentiell allen das Attribut «Spitzenmedizer» zukommt und sich somit die gesamte Ärzteschaft in

\section{Mit unspektakulären Massnahmen erziele die Hausarztmedizin grosse Effekte - rein epidemiologisch gesehen hätten hausarztbasierte Interventionen weit mehr Impact als hochtechnisierte Eingriffe.}

- Spitzenmedizin kann auch qualitativ definiert werden, wie dies am Symposium unter anderen Prof. Paul Mohacsi, leitender Kardiologe am Inselspital Bern, tat. Für ihn sind wesentliche Kennzeichen der Spitzenmedizin Patientenzentriertheit, hohes fachliches Niveau und Interdisziplinarität.

- Der Begriff «Spitzenmedizin», so vermutete Ludwig Hasler, sei wohl eine mediale Erfindung, um die Art von Medizin zu bezeichnen, mit der man Aufsehen erregen, Sensationen erzeugen könne. Er hielt einen umfassenden Anspruch dagegen: So wie sich der Spitzenkoch nicht dadurch auszeichne, dass er 100 Kalbslebern pro Tag zubereite, sondern sich bei Fleisch, Fisch, Gemüse,

\section{Abschied und Neuanfang}

Ein SGIM Platin-Symposium ohne Werner Bauer? Eigentlich undenkbar, aber man wird sich mit dieser Perspektive arrangieren müssen - zumindest, was seine Rolle als treibende Kraft und Gastgeber betrifft. Aufgrund seiner neuen Aufgabe als Präsident des Schweizerischen Instituts für Weiter- und Fortbildung SIWF, die er im Juni dieses Jahres übernommen hat, ist der Past President der Schweizerischen Gesellschaft für Innere Medizin aus den SGIM-Führungsgremien zurückgetreten und wird damit auch nicht mehr für die Organisation des Platin-Symposiums zur Verfügung stehen.

Dieses Symposium, das in einzigartiger Weise einmal im Jahr Vertreterinnen und Vertreter aus Medizin, Politik, Wirtschaft und Kultur für 24 Stunden zu einem intensiven Austausch zusammenbringt, ist unzweifelhaft Werner Bauers Kind. Seit der ersten Durchführung im Jahr 2005 war er Seele und Motor des Anlasses, auch wenn an dieser einem Topf wiederfindet, werden Diskussionen, die Unterschiede herausarbeiten wollen, rein begrifflich schwierig.

Die Frage, welche Art von Hausarztmedizin die Spitzenmedizin brauche und umgekehrt (um sich optimal in den Dienst der Patienten stellen zu können, wäre hier wohl zu ergänzen), setzt die Berechtigung beider Ausprägungen der ärztlichen Tätigkeit voraus. Dass es sowohl die hausärztliche als auch die hochspezialisierte Medizin braucht, wurde denn am Symposium auch nicht in Zweifel gezogen. Dagegen waren durchaus unterschiedliche Positionen auszumachen, wenn es um den Stellenwert und das Verhältnis von Hausarzt- und Spitzenmedizin ging.

Stelle die zahlreichen Helferinnen und Helfer im Hinter- und teilweise auch im Vordergrund nicht vergessen werden sollen. Doch was SGIM-Präsident Ueli Bürgi, gemeinsam mit SGIM-Vizepräsident Jürg Pfisterer auch in diesem Jahr Co-Gastgeber des Symposiums, einst mit dem ihm eigenen trockenen Humor formulierte, war mehr als nur ein rhetorischer Gag: «Werner Bauer macht, und wir finden es gut.»

Wer Werner Bauer kennt, ahnt schon, dass er wohl auch in seiner neuen Funktion wieder «irgendetwas» auf die Beine stellen wird. Aber auch Platin-Begeisterten braucht nicht bange zu sein. Die SGIM wird das Symposium im Jahr 2011 erneut durchführen. Bevor nun bereits neue Erwartungen formuliert werden, sei Werner Bauer und dem «Platin-Team» aber eine Verschnaufpause gegönnt und an dieser Stelle herzlich für sechs wunderbare Symposien gedankt.

Bruno Kesseli 
Für einige überraschend, stellte Thomas Rosemann, Direktor des Instituts für Hausarztmedizin, in seinem Referat nicht die besondere Befähigung der Hausärztinnen und Hausärzte zur individuellen «Rundumbetreuung» ihrer Patientinnen und Patienten in den Vordergrund. Vielmehr argumentierte er aus einer epidemiologischen Perspektive: Mit unspektakulären Massnahmen erziele die Hausarztmedizin grosse Effekte - rein epidemiologisch gesehen hätten hausarztbasierte Interventionen weit mehr Impact als hochtechnisierte Eingriffe. Gesundheitsökonomisch sei somit der Return on investment in der Hausarztmedizin am grössten.

Die Kunst der Hausärzte liegt gewissermassen im virtuosen Umgang mit dem Bayes'schen Theorem*, lus, will Ergebnisse verbessern (führt aber nicht immer zu besseren Ergebnissen) - und sie braucht die Hausarztmedizin.

Bei der Beantwortung der Frage, warum die Spitzenmedizin die Hausarztmedizin braucht, traf sich Falk mit dem (nach ihm sprechenden) Rosemann. Auf die gesamte Bevölkerung bezogen, brauchen wenige Patientinnen und Patienten die Spitzenmedizin, aber sehr viele brauchen eine Hausärztin oder einen Hausarzt - eine Entwicklung, die sich aufgrund der demographischen Entwicklung noch zuspitzen wird. Und auch in Fällen, bei denen von der Problematik her eine hochspezialisierte Intervention in Frage kommt, kann die hausärztliche Unterstützung bei der Indikationsstellung unabdingbar sein. Denn, wie Falk am

\section{Spitzenmedizin braucht Spitzentechnologie, Interdisziplinarität und Visionen - und sie braucht die Hausarztmedizin.}

* Das Bayes'sche Theorem, benannt nach dem Mathematiker Thomas Bayes, gibt an, wie man mit sogenannt bedingten Wahrscheinlichkeiten rechnet. In der Medizin ist das Theorem im Zusammenhang mit der durch Sensitivität und Spezifität gekennzeichneten «Leistungsfähigkeit» von Tests von Bedeutung.

** Die Gesellschaft für Thoraxchirurgie der USA (Society of Thoracic Surgery) hat ein Instrument zur Abschätzung des Mortalitätsrisikos sowie weiterer Risiken im Zusammenhang mit operativen (Thorax-) Eingriffen entwickelt (z.B. lange Hospitalisationsdauer Nierenversagen). Der «STS Risk Calculator» ist unter www.sts.org/sections/ stsnationaldatabase/ riskcalculator/ auch online zugänglich. oder wie Rosemann es formulierte: Die Herausforderung der hausärztlichen Tätigkeit ist das Niedrigprävalenzsetting. Es sind die Hausärztinnen und Hausärzte, die aus den 100 Patienten mit Brustschmerzen denjenigen erkennen und an die Universitätsklinik überweisen müssen, bei dem ein Herzinfarkt vorliegt und der einer sofortigen «spitzenmedizinischen» Intervention bedarf. Die Hausarztmedizin, so Rosemann, liefert durch gezielte Selektion erst die Grundlage für eine effiziente Spitzenmedizin.

\section{Hochspezialisierte Medizin braucht Hausarzt- medizin}

Für Volkmar Falk ist Spitzenmedizin immer (auch) Pionierarbeit. Der Direktor der Klinik für Herz- und Gefässchirurgie am Universitätsspital Zürich stützte diese These mit einer Reihe eindrücklicher Beispiele aus der Geschichte der Medizin: etwa mit der ersten erfolgreichen Herznaht nach Stichverletzung durch Ludwig Rehn im Jahr 1896, der weltweit ersten Herzschrittmacherimplantation durch Ake Senning im Jahr 1958 oder der Erfindung des Ballonkatheters durch Andreas Grüntzig in den 1970er Jahren, die er als Beispiel für eine «konkurrierende «disruptive Innovation» anführte.

Die durch die neue Technik «bedrohten» Chirurgen hätten das Verfahren erst einmal mit Einschätzungen wie «lächerlich», «gefährlich», «unethisch», «ein Witz» oder «geht nicht» bedacht. Ein fast schon gesetzmässiger Vorgang im Zusammenhang mit disruptiver Innovation, die nach Falk ein Merkmal der Spitzenmedizin ist. Weitere Bestandteile des Mosaiks aus seiner Sicht: Spitzenmedizin baut auf der Arbeit anderer auf, sie braucht Spitzentechnologie, Interdisziplinarität und Visionen, wird durch Innovationsdruck gefördert, unterliegt dem Produktelebenszyk-
Beispiel zweier 90-jähriger Patientinnen zeigte, selbst bei gleichem Alter und identischem STS-Risiko** kann im einen Fall eine Operation sinnvoll sein, im andern nicht. Hier kommen Begriffe wie Gebrechlichkeit («Frailty») ins Spiel. Für die mag es zwar mittlerweile eigene Indizes geben, aber wie meinte Ludwig Hasler treffend: «Der Hausarzt kennt nicht alle Methoden zur Wiederherstellung von Sonderorganen. Er kennt aber die Geschichte des Patienten. Er kann Störungen lesen wie Schlüsselstellen in einem Roman.» $\mathrm{Zu}$ dieser hausärztlichen Kompetenz scheint es bis auf weiteres keine Alternativen zu geben.

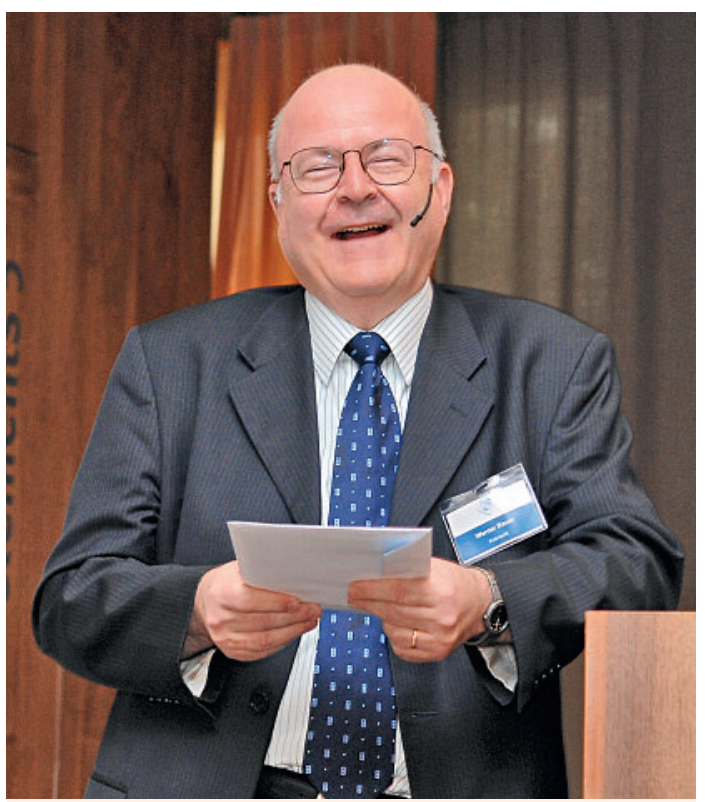

Hatte auch am letzten Platin-Symposium unter seiner Ägide Grund zum Lachen: Werner Bauer. 


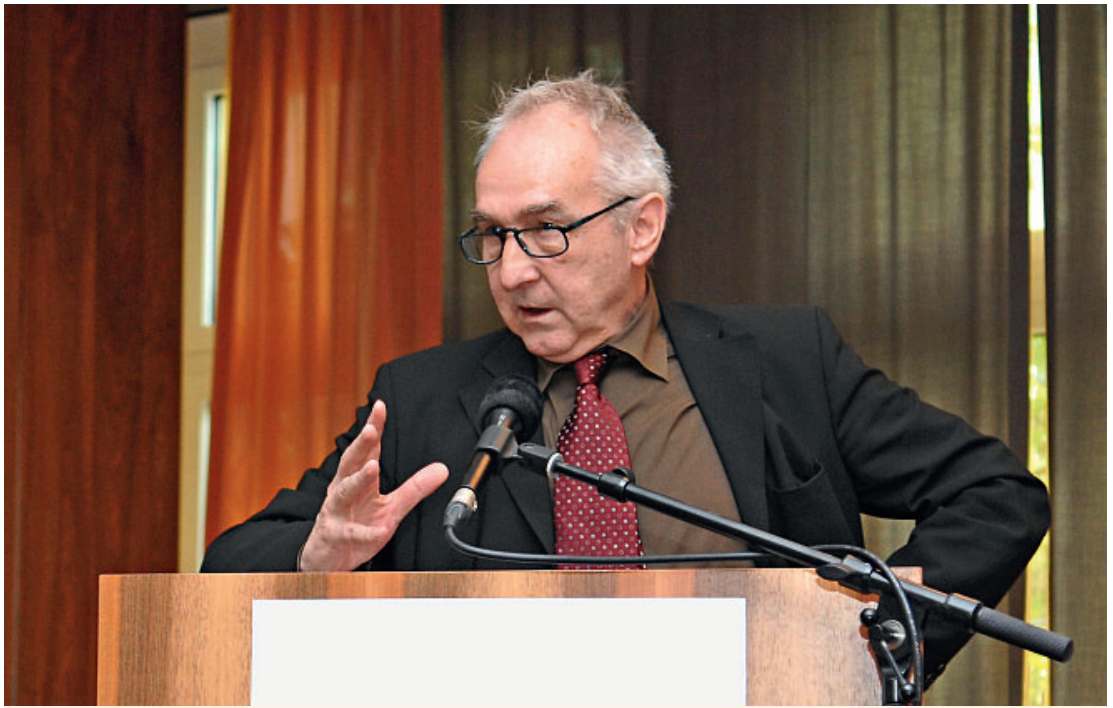

«Störungen lesen wie Schlüsselstellen in einem Roman»: Ludwig Hasler, Philosoph und Publizist.

\section{Erfolgreiche Raufereien}

Moderator Iwan Rickenbacher griff prägnante Statements einzelner Referenten in den Podiumsdiskussionen geschickt auf und unternahm zumindest den Versuch, die Bildung von Thesen zum Symposiumsthema anzuregen. Beim Stichwort «Zusammenraufen» konnte Marc Müller, Präsident der «Hausärzte Schweiz», immerhin die positive Bilanz ziehen, dass in seinem Bereich interne Raufereien mittlerweile mit gutem Ergebnis - beispielsweise dem Facharzttitel Allgemeine Innere Medizin» - abgeschlossen werden konnten. Peter Wiedersheim, Präsident der Konferenz der Ostschweizer Ärztegesellschaften und der Ärztegesellschaft des Kantons St. Gallen, wies auf den Erfolg hin, der durch den Zusammenschluss der Ärzteschaft über die Kantonsgrenzen hinweg, etwa in Bezug auf einen einheitlichen Taxpunktwert für die Region Ostschweiz, erzielt werden konnte.

Versorgungsregionen werden inskünftig ein wichtiges Instrument zur Sicherung eines qualitativ guten, finanzierbaren Gesundheitswesens sein, wie auf dem Podium nicht nur Ständerätin Christine EgerszegiObrist betonte. Die Politikerin sah - wohl für einige überraschend - kein Primat der Politik bei der Organisation der medizinischen Versorgung: «Die Entwicklungen müssen von unten kommen, die Politik kann dann allenfalls die Spielregeln festlegen.» Marc Müller traute den Hausärzten zu, die Steuerung des Systems zu übernehmen, wenn die Rahmenbedingungen stimmen, während für Peter Wiedersheim

\section{«Die Entwicklungen müssen von unten kommen, die Politik kann dann allenfalls die Spielregeln festlegen.»}

stärker kritisch hinterfragen lassen, wenn es um Fragen der Verteilung knapper Ressourcen geht, mit denen sich der Philosoph Richard Egger in seinem Referat befasste.

Dass sich die Verantwortungs- und Entscheidungsträger mit solchen Fragen schwertun, zeigte Peter Suter, Präsident der Schweizerischen Akademie der medizinischen Wissenschaften, der unter dem Titel «Spektakel oder ernste Sache?» zum Thema Koordination und Konzentration der hochspezialisierten Medizin sprach. Ob es gelingt, dass sich die wichtigen Interessengruppen auch innerhalb der Ärzteschaft zusammenraufen, wie er dies forderte, damit bestmögliche und sachliche richtige Lösungen durchkommen und Resultate und Qualität über falschem politischem und medizinischem Prestigedenken stehen - niemand vermag es zurzeit zu sagen. zunächst grundlegende Fragen zu klären wären. Wenn von Kosten gesprochen werde, sei meist unklar, was darunter zu subsumieren sei. Es fehle die volkswirtschaftliche Gesamtkostenbetrachtung. Auch in Bezug auf die Messgrössen ortet er hierzulande Defizite, die allerdings in Deutschland und Österreich genauso gegeben seien, wie er im Rahmen der von ihm mitorganisierten Gesundheitspiazza mit den zwei Nachbarländern festgestellt habe.

Iwan Rickenbacher konnte zum Schluss zwar keine ausformulierten Thesen präsentieren, aber immerhin die Bilanz ziehen, dass es gelungen war, «Handlungsspielräume zu definieren». Diese auszufüllen, liegt nun an den Podiumsteilnehmerinnen und -teilnehmern aus Medizin, Politik, Wirtschaft und Kultur. Impulse haben sie am 6. SGIM PlatinSymposium reichlich erhalten. 